\title{
Association between the National Health Insurance coverage benefit extension policy and clinical outcomes of ventilated patients: a retrospective study
}

\author{
Wanho Yoo', Saerom Kim¹, Soohan Kim', Eunsuk Jeong ${ }^{1}$, Kwangha Lee ${ }^{1,2}$ \\ ${ }^{1}$ Division of Pulmonary, Allergy, and Critical Care Medicine, Department of Internal Medicine, Pusan National University Hospital, Busan; ${ }^{2}$ Department of Internal \\ Medicine, Pusan National University School of Medicine, Busan, Korea
}

Background: This study aimed to investigate the association between the Korean National Health Insurance coverage benefit extension policy and clinical outcomes of patients who were ventilated owing to various respiratory diseases.

Methods: Data from 515 patients (male, 69.7\%; mean age, 69.8 \pm 12.1 years; in-hospital mortality rate, $28.3 \%$ ) who were hospitalized in a respiratory intensive care unit were retrospectively analyzed over 5 years.

Results: Of total enrolled patients, 356 (69.1\%) had one benefit items under this policy during their hospital stay. They had significantly higher medical expenditure (total: median, 23,683 vs. 12,742 U.S. dollars [USD], $\mathrm{P}<0.001$ ), out-of-pocket (median, 5,932 vs. 4,081 USD; $\mathrm{P}<0.001$ ), and a lower percentage of out-of-pocket medical expenditure relative to total medical expenditure (median, $26.0 \%$ vs. $32.2 \%$; $P<0.001)$. Patients without benefit items associated with higher in-hospital mortality (hazard ratio [HR], 2.794; 95\% confidence interval [Cl], 1.980-3.941; $P<0.001$ ). In analysis of patients with benefit items, patients with three items ("cancer," "tuberculosis," and "disability") had significantly lower out-of-pocket medical expenditure (3,441 vs. 6,517 USD, P<0.001), and a lower percentage of out-of-pocket medical expenditure relative to total medical expenditure (17.2\% vs. $27.7 \%, P<0.001)$. They were associated with higher in-hospital mortality (HR, 3.904; 95\% Cl, 2.533-6.039; $\mathrm{P}<0.001)$.

Conclusions: Our study showed patients with benefit items had more medical resources and associated improved in-hospital survival. Patients with the aforementioned three benefit items had lower out-of-pocket medical expenditure due to the implementation of this policy, but higher in-hospital mortality.

Key Words: health expenditures; health insurance; mechanical ventilators; mortality

\section{INTRODUCTION}

Patients receiving mechanical ventilation (MV) require various medical resources for a long period of time in an intensive care unit (ICU). In addition, advances in the management of acute critical care intervention have led to improvement of survival, which is associated with

\section{Original Article}

Received: October 5, 2021

Revised: January 24, 2022

Accepted: January 27, 2022

\section{Corresponding author}

Kwangha Lee

Division of Pulmonary, Allergy, and

Critical Care Medicine, Department

of Internal Medicine, Pusan National

University School of Medicine, 179

Gudeok-ro, Seo-gu, Busan 49241,

Korea

Tel: +82-51-240-7743

Fax: +82-52-245-3127

E-mail: jubilate@pusan.ac.kr
Copyright (C) 2022 The Korean Society of Critical Care Medicine

This is an Open Access article distributed under the terms of Creative Attributions Non-Commercial License (https:// creativecommons.org/li-censes/by-nc/4.0/ which permits unrestricted noncommercial use, distribution, and reproduction in any medium, provided the original work is properly cited. 
high medical costs. As a result, high out-of-pocket medical expenditures place a severe financial burden on the families or surrogates of patients.

To address the financial burden of healthcare, the National Health Insurance (NHI) System was implemented in the Republic of Korea (hereafter called Korea) in 1977 [1]. In this system, the NHI program is a social insurance benefit scheme that covers the whole population [1-3]. In addition to this insurance program, the Korean government has introduced a health insurance benefit extension policy targeting the elderly, pregnant women and newborns, Medicare recipients, and individuals with severe illnesses, malignancies, and rare incurable diseases for payment relief [4]. The benefit items and associated benefit rules under this policy have been implemented, including extensions of benefit period, and improvements of the medical reimbursement systems. This policy has been expanded to provide medical assistance without causing patients severe financial difficulties.

As a result of improvements in critical care medicine, many patients receiving ventilator care require various medical resources for a long time in an ICU, which is associated with high medical expenditures. In the present study, we hypothesized that the expansion of benefit items and revision of associated rules would be associated with beneficial outcomes for patients requiring $\mathrm{MV}$, and that the clinical courses and outcomes of these patients would differ according to the various benefit items under this policy. In literature, however, few studies have investigated associations between the NHI benefit extension policy and clinical outcomes of critically ill Korean patients. Therefore, the present study aimed to investigate the current status of benefit items under this policy and the association between these benefit items and clinical outcomes of critically ill NHI beneficiaries who received ventilator care owing to various respiratory diseases.

\section{MATERIALS AND METHODS}

\section{Study Design and Patient Selection}

This retrospective, observational study was conducted in the 12-bed adult respiratory ICU of a regional center for respiratory diseases that was established in December 2015. This center is located in a 1,200-bed university-affiliated tertiary care hospital. The respiratory ICU has full cardiovascular facilities and close airway monitoring equipment, with the nurse-tobed ratio is 1:3. All subjects were managed according to therapeutic recommendations based on a lung-protective ventila-

\section{KEY MESSAGES}

- The Korean National Health Insurance benefit extension policy was associated with lower in-hospital mortality of National Health Insurance beneficiaries requiring ventilator care.

- In analysis of patients with benefit items, patients with three items ("cancer," "tuberculosis," and "disability") had a significantly lower percentage of out-of-pocket medical expenditure relative to total medical expenditure, but higher in-hospital mortality.

tor strategy [5].

Data from patients who were admitted to the respiratory ICU between December 1, 2015, and November 30, 2020, were retrospectively evaluated. The survival status of all patients was obtained until March 10, 2021. All adult subjects ( $\geq 18$ years of age) admitted to ICU were assessed for eligibility. Patients who required ventilator care for more than 24 hours and who required ventilator care primarily due to respiratory diseases were included. Medical Aid (MA) beneficiaries were excluded because MA provides healthcare benefits to low-income families and sets an upper limit for out-of-pocket medical expenditure based on the National Basic Living Security Act [6,7]; the NHI benefit extension policy would be little effect on clinical outcomes in these patients. Patients were also excluded if they had not received ventilator care, had other minor types of insurance such as automobile insurance and industrial accident compensation insurance, had no insurance, or information about their insurance was lacking. Private insurance programs were not analyzed in this study (Figure 1).

The relevant medical, laboratory, and radiologic data were extracted and used to fill out a case report form for each patient. These data were then analyzed. The study protocol was approved by the Institutional Review Board of Pusan National University Hospital (IRB No. 2104-010-101). Owing to the observational nature of the study, the need for informed consent from enrolled subjects or their families or surrogates was waived. This study had no impact on the treatment of the enrolled patients.

\section{Data Collection}

Demographic and clinical data, including age, sex, body mass index, length of stay (LOS) in the ICU and hospital, and duration of MV, were retrospectively obtained from the electronic medical records (EMRs) of each subject. Illness severity was 


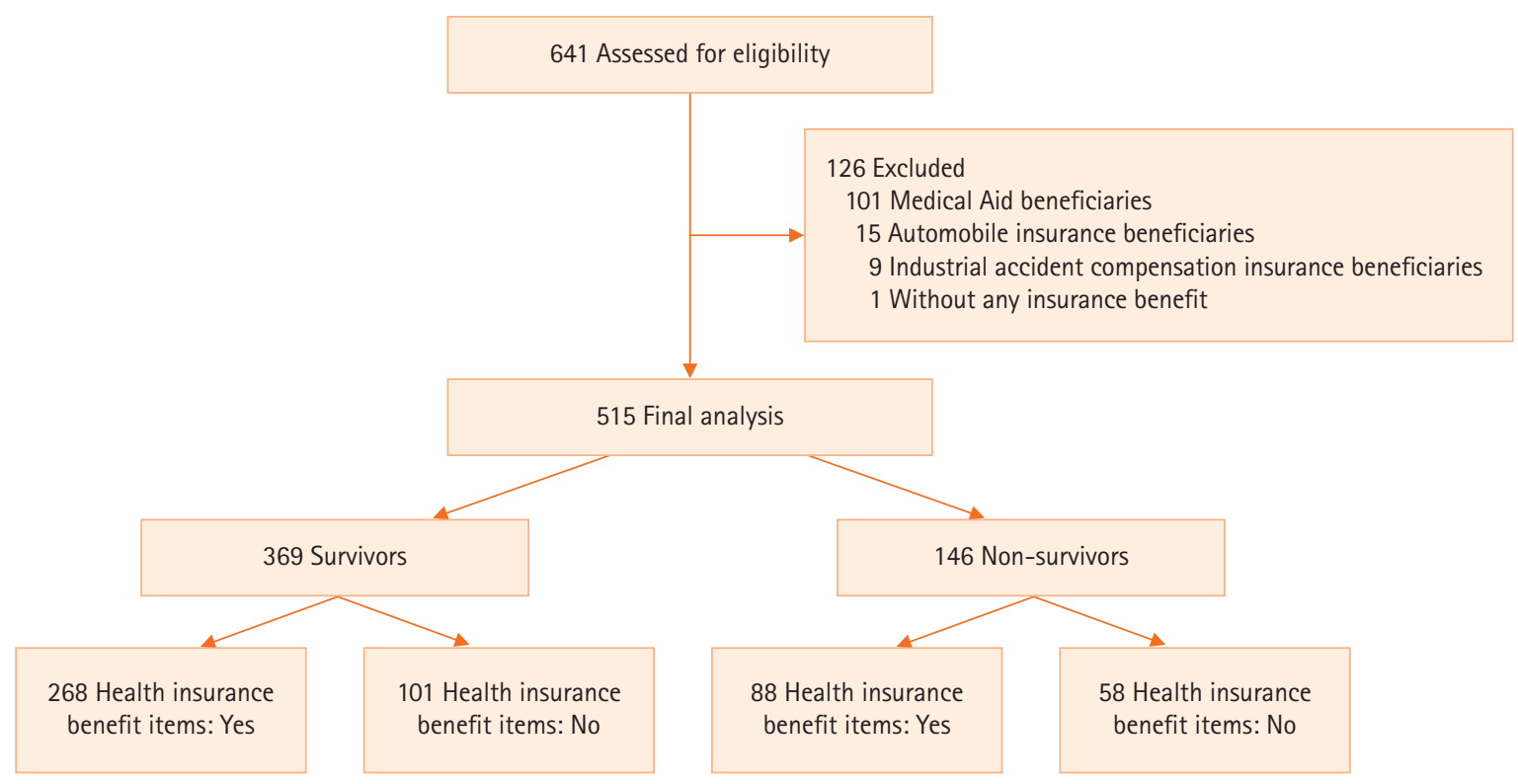

Figure 1. Flowchart of patient selection and clinical course.

measured using the Acute Physiology and Chronic Health Evaluation (APACHE) II score, and accompanying organ failure was assessed using the Sequential Organ Failure Assessment (SOFA) score [8,9]. The APACHE II and SOFA scores were calculated from laboratory and clinical data obtained within the first 24 hours of ICU admission. On admission, the underlying comorbidities of all enrolled subjects were gathered from EMRs. In addition, to determine concurrent comorbidities prior to admission, Charlson's weighted index of comorbidities (WIC) was evaluated from EMRs [10]. The primary reason for ventilator care at the time of ICU admission, and whether attending physicians discussed life-sustaining therapy (withdrawing or withholding ICU care) with patients' families or surrogates were also evaluated from the medical records. ICU and in-hospital mortality after ICU admission was assessed. The primary outcome measure was in-hospital mortality.

To evaluate the association between medical expenditure per patient and clinical outcomes, total and out-of-pocket medical expenditure for all medical resources (including all medicines) during the hospital (ICU and general ward) stay was retrieved from the EMRs of each subject with the permission of the IRB. Expenditure is presented in U.S. dollars (USD) using an exchange rate of 1 USD equaling 1,140.10 Korean won (exchange rate on March 10, 2021). Also, the main applicable benefit items under the NHI benefit extension policy to reduce each patient's out-of-pocket medical expenditure during their hospital stay were evaluated.

\section{Statistical Analysis}

Continuous variables are expressed as the mean \pm standard deviation (SD) if they had a normal distribution, or median with interquartile range. The Student t-test and Wilcox ranksum test were used to compare continuous variables with a normal distribution and variables with a non-normal distribution, respectively. Categorical variables are expressed as numbers (percentages) and were compared using the chisquare test or Fisher's exact test (for small numbers), as applicable. Pearson's correlation coefficient $(\gamma)$ was calculated to evaluate the relationship between medical expenditure (total and out-of-pocket) per person and LOS (in the hospital and ICU. Univariate proportional hazard models were developed to determine the relationship between healthcare insurance benefit items and in-hospital mortality. Variables with $\mathrm{P}<0.10$ were included in multivariate Cox proportional hazard models. Hazard ratios with $95 \%$ confidence intervals are reported. Kaplan-Meier estimates of in-hospital mortality were stratified according to health insurance benefit items status, and curves were compared using log-rank test. All tests were two-tailed, and P-values $<0.05$ were considered statistically significant. All analyses were performed using the language $\mathrm{R}$ (http://cran.r-project.org) version 4.0.5, and additional packages (survival). 


\section{RESULTS}

\section{Characteristics of Total Patients}

During the study period, 924 patients were admitted to respiratory ICU. After application of exclusion criteria, 515 patients were eligible for participation (Figure 1). Their ICU and in-hospital mortality rate was $22.9 \%$ and $28.3 \%$, respectively. The most common diagnosis leading to MV was pneumonia (87.2\%). Chronic lung diseases (such as chronic obstructive pulmonary disease, interstitial lung disease, and destroyed lung caused by various origins) were the most common underlying diseases. The median total and out-of-pocket medical expenditures during hospital stays were 19,986 (range, 2,560-573,591) and 5,319 (range, 703-134,199) USD, respectively. The median percentage of out-of-pocket medical expenditure relative to total medical expenditure was $28.3 \%$ (range, 8.3\%-86.3\%). There was a positive correlation between medical expenditure (total and out-of-pocket) and hospital LOS $(\gamma=0.944, \mathrm{P}<0.001$ and $\gamma=0.875, \mathrm{P}<0.001$, respectively). In total, 356 (69.1\%) of NHI beneficiaries had benefit items under the NHI benefit extension policy.

\section{Comparisons between Patients with and without Benefit} Items under Health Insurance Benefit Extension Policy The clinical characteristics of patients with and without benefit items are compared in Table 1. Patients with benefit items had a significantly longer hospital and ICU LOS, higher medical expenditure (total and out-of-pocket), a lower percentage of out-

Table 1. Clinical characteristics of total patients and comparisons between with and without National Health Insurance benefit items

\begin{tabular}{|c|c|c|c|c|}
\hline \multirow{2}{*}{ Variable } & \multirow{2}{*}{ Total $(n=515)$} & \multicolumn{2}{|c|}{ Health insurance benefit item } & \multirow{2}{*}{ P-value } \\
\hline & & Yes $(n=356)$ & No $(n=159)$ & \\
\hline Male & $359(69.7)$ & $256(71.9)$ & $103(64.8)$ & 0.128 \\
\hline Age (yr) & $69.8 \pm 12.1$ & $69.3 \pm 12.2$ & $70.9 \pm 11.8$ & 0.149 \\
\hline $\mathrm{BMI}\left(\mathrm{kg} / \mathrm{m}^{2}\right)$ & $21.5 \pm 4.8$ & $21.6 \pm 4.6$ & $21.2 \pm 4.9$ & 0.356 \\
\hline ICU LOS (day) & $11(6-18)$ & $12(7-19)$ & $8(5-15)$ & $<0.001$ \\
\hline MV LOS (day) & $9(4-18)$ & $11(5-19)$ & $6(3-14)$ & $<0.001$ \\
\hline Hospital LOS (day) & $25(13-45)$ & $31(19-53)$ & $13(7-24)$ & $<0.001$ \\
\hline APACHE II score ${ }^{b}$ & $21.6 \pm 6.7$ & $21.5 \pm 6.4$ & $21.6 \pm 7.4$ & 0.893 \\
\hline SOFA score ${ }^{b}$ & $7.7 \pm 3.1$ & $7.6 \pm 2.9$ & $8.1 \pm 3.5$ & 0.111 \\
\hline Main diagnosis leading to MV: pneumonia & $449(87.2)$ & 309 (86.8) & $140(88.1)$ & 0.802 \\
\hline \multicolumn{5}{|l|}{ Comorbidity } \\
\hline Chronic lung disease & $196(38.1)$ & $137(38.5)$ & $59(37.1)$ & 0.842 \\
\hline Diabetes & $175(34.0)$ & $127(35.7)$ & $48(30.2)$ & 0.266 \\
\hline Cardiovascular disease & $163(31.7)$ & $111(31.2)$ & $52(32.7)$ & 0.809 \\
\hline Chronic neurological disease & $152(29.5)$ & $104(29.2)$ & $48(30.2)$ & 0.905 \\
\hline Solid malignant tumor & $96(18.6)$ & 77 (21.6) & 19 (11.9) & 0.013 \\
\hline Chronic kidney disease & $57(11.1)$ & 45 (12.6) & $12(7.5)$ & 0.121 \\
\hline Connective tissue disease & $38(7.4)$ & $34(9.6)$ & $4(2.5)$ & 0.003 \\
\hline Chronic liver disease & $23(4.5)$ & $15(4.2)$ & $8(5.0)$ & 0.854 \\
\hline Hematological malignancy & $5(1.0)$ & $4(1.1)$ & $1(0.6)$ & $>0.999$ \\
\hline Total medical expenditures (USD) ${ }^{c}$ & $19,986(11,959-32,104)$ & $23,683(15,098-36,597)$ & $12,742(8,790-22,118)$ & $<0.001$ \\
\hline Out-of-pocket medical expenditures (USD) ${ }^{c}$ & $5,319(3,136-8,488)$ & $5,932(3,421-8,801)$ & $4,081(2,717-7,268)$ & $<0.001$ \\
\hline Ratio of out-of-pocket for total medical expenditures (\%) & $28.3(22.0-32.6)$ & $26.0(18.8-30.2)$ & $32.2(28.6-35.9)$ & $<0.001$ \\
\hline Tracheostomy at hospital discharge & $308(59.8)$ & $238(66.9)$ & $70(44.0)$ & $<0.001$ \\
\hline $\begin{array}{l}\text { Discussion with attending physicians about withdrawing or } \\
\text { withholding life-sustaining treatment }\end{array}$ & $168(32.6)$ & $99(27.8)$ & $69(43.8)$ & $<0.001$ \\
\hline In-hospital mortality & $146(28.3)$ & $88(24.7)$ & $58(36.5)$ & 0.009 \\
\hline
\end{tabular}

Values are presented as number (\%), mean \pm standard deviation, or median (interquartile range).

BMI: body mass index; ICU: intensive care unit; LOS: length of stay; MV: mechanical ventilation; APACHE: Acute Physiology and Chronic Health Evaluation; SOFA: Sequential Organ Failure Assessment; USD: U.S. dollars.

${ }^{a}$ Comparisons between patients with and without health insurance benefit items; ${ }^{b}$ All clinical data were calculated or obtained from medical records on the day of ICU admission; 'Total and out-of-pocket medical expenditure for all medical resources used (including all medicines) during ICU and general ward stays was retrieved. 
of-pocket medical expenditure relative to total medical expenditure, and lower in-hospital mortality than patients without benefit items.

\section{Comparisons of Clinical Outcomes According to Benefit Items under Health Insurance Benefit Extension Policy} Nine benefit items were applied to NHI beneficiaries, and the most common benefit item was "long-term hospitalization" (Figure 2). Patients with three benefit items ("cancer," "tuberculosis," and "disability" categorized as group A) had significantly higher in-hospital mortality than patients with other benefit items (categorized as group B). Patients with these benefit items had significantly higher APACHE II and SOFA scores, a higher Charlson's WIC, and a lower percentage of out-of-pocket medical expenditure relative to total medical expenditure. Furthermore, families or surrogates of patients with these benefit items had significantly more discussions with their attending physicians about life-sustaining therapy (Table 2).

\section{Association between Healthcare Insurance Benefit Items and In-hospital Mortality}

Univariate and multivariate Cox regression were constructed to evaluate the association between healthcare insurance ben- efit items and in-hospital mortality in total enrolled patients (Table 3). We found patients without benefit items were significantly associated with higher in-hospital mortality. The Kaplan-Meier estimate of survival was consistent with a survival benefit of benefit items (Figure 3A). In further analysis of patients with benefit items, Group A showed a significant association with higher in-hospital mortality. Also, the Kaplan-Meier estimate of survival was consistent with a survival benefit of group B (Figure 3B).

\section{DISCUSSION}

The present study evaluated the association between the NHI benefit extension policy and clinical outcomes of NHI beneficiaries requiring ventilator care owing to respiratory diseases. Furthermore, we investigated the differences of clinical outcomes according to various benefit items. To our knowledge, this is the first study to analyze the clinical characteristics and outcomes of ventilated patients under this policy in Korea.

We found that about $70 \%$ of total enrolled patients were eligible for one benefit during their hospital stay, and these patients used more medical resources, reflected by total medical expenditures, and their in-hospital mortality was lower than that of patients who were ineligible for benefits. Our findings

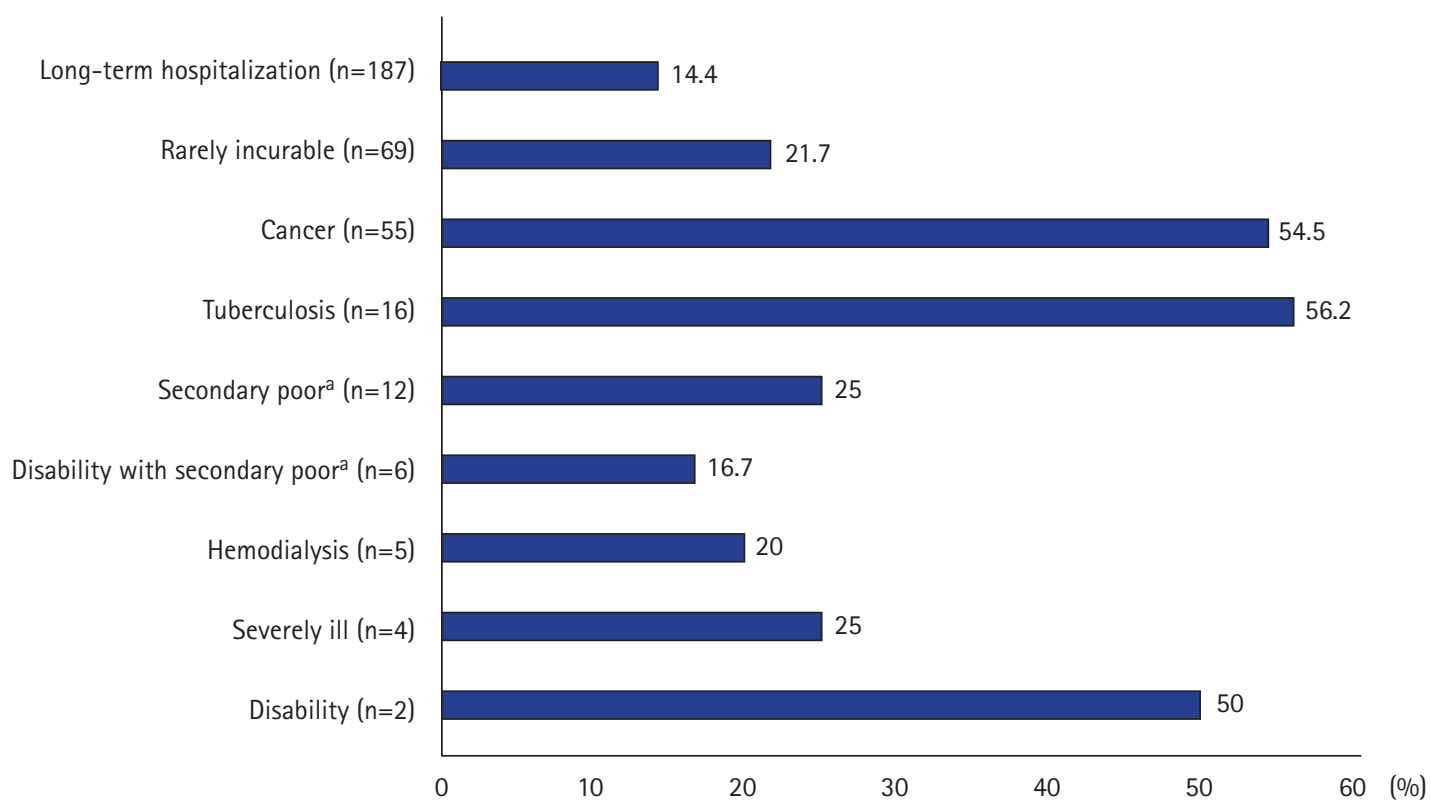

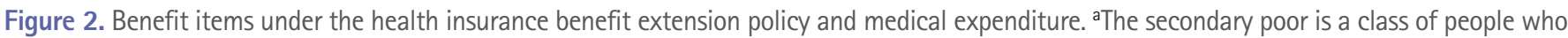
are economically similar to or slightly better off than the poor. They are potentially poor, but cannot receive some benefits available to Medical Aid beneficiaries because their income exceeds the minimum cost of living due to their ability to work and they have some fixed property. 
Table 2. Comparison of clinical characteristics according to benefit items under health insurance benefit extension policy

\begin{tabular}{|c|c|c|c|}
\hline \multirow{2}{*}{ Variable } & \multicolumn{2}{|c|}{ Health insurance benefit item } & \multirow{2}{*}{ P-value } \\
\hline & Group A (n=73) & Group B ( $n=283)$ & \\
\hline Male & $59(80.8)$ & $197(69.6)$ & 0.079 \\
\hline Age $\geq 65 \mathrm{yr}$ & $49(67.1)$ & $217(76.7)$ & 0.128 \\
\hline APACHE II score ${ }^{a}$ & $22.9 \pm 6.8$ & $21.2 \pm 6.3$ & 0.060 \\
\hline SOFA score ${ }^{a}$ & $8.6 \pm 2.9$ & $7.3 \pm 2.9$ & 0.001 \\
\hline $\mathrm{BMI}\left(\mathrm{kg} / \mathrm{m}^{2}\right)$ & $21.1 \pm 3.7$ & $21.8 \pm 4.3$ & 0.146 \\
\hline Main diagnosis leading to MV: pneumonia & $58(79.5)$ & $251(88.7)$ & 0.059 \\
\hline Charlson's WIC & $3.4 \pm 1.8$ & $2.7 \pm 1.8$ & 0.006 \\
\hline Tracheostomy at hospital discharge & $39(53.4)$ & 199 (70.3) & 0.009 \\
\hline ICU LOS & $11(5-19)$ & $12(8-19)$ & 0.117 \\
\hline MV LOS & $11(4-18)$ & $11(5-19)$ & 0.334 \\
\hline Hospital LOS & $24(13-54)$ & $33(21-53)$ & 0.010 \\
\hline Total medical expenditure (USD) ${ }^{b}$ & $21,036(12,554-34,026)$ & $23,772(15,446-36,622)$ & 0.327 \\
\hline Out-of-pocket medical expenditure (USD) ${ }^{b}$ & $3,441(2,254-7,139)$ & $6,517(4,263-9,602)$ & $<0.001$ \\
\hline Out-of-pocket medical expenditure relative to total medical expenditure (\%) & $17.2(13.7-21.0)$ & $27.7(21.9-31.1)$ & $<0.001$ \\
\hline $\begin{array}{l}\text { Discussion with attending physicians about withdrawing or withholding } \\
\text { life-sustaining treatment }\end{array}$ & $41(56.2)$ & $58(20.5)$ & $<0.001$ \\
\hline In-hospital mortality & $40(54.8)$ & $48(17.0)$ & $<0.001$ \\
\hline
\end{tabular}

Values are presented as number (\%), mean \pm standard deviation, or median (interquartile range). Group A includes following benefit items: cancer, tuberculosis and disability. Group B includes following benefit items: long-term hospitalization, rarely incurable, secondary poor, secondary poor with disability, hemodialysis, and severely ill.

APACHE: Acute Physiology and Chronic Health Evaluation; SOFA: Sequential Organ Failure Assessment; BMI: body mass index; MV: mechanical ventilation; WIC: weighted index of comorbidities; ICU: intensive care unit; LOS: length of stay; USD: U.S. dollars.

${ }^{a}$ All clinical data were calculated or obtained from medical records on day of ICU admission; ${ }^{b}$ Total and out-of-pocket medical expenditures for all medical resources used (including all medicines) during ICU and general ward stay were retrieved.
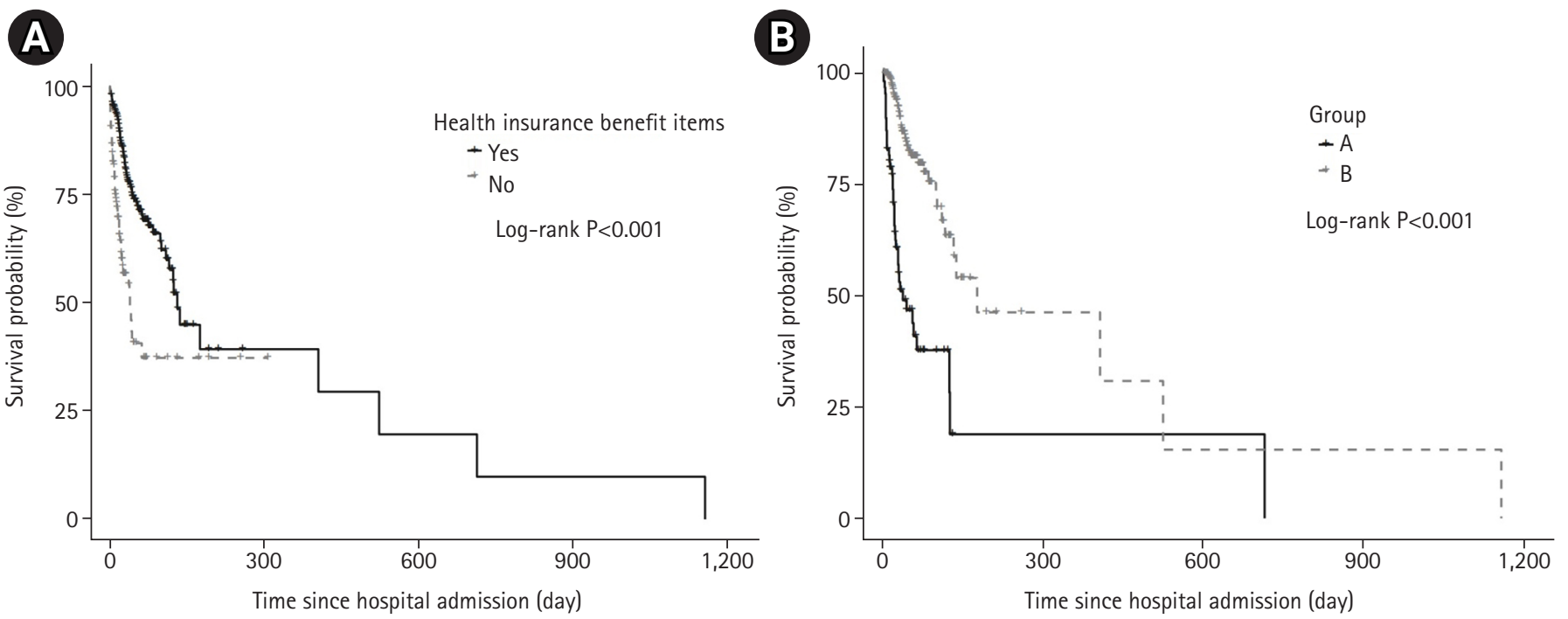

Figure 3. Kaplan-Meier curves of survival curves of in-hospital survival after intensive care unit admission according to the presence or absence of health insurance benefit items in total enrolled patients (Log-rank, 37.7; $P<0.001)(A)$ and according to categorized as group $A$ or $B$ in patients with health insurance benefit items (Log-rank, 46.3; $\mathrm{P}<0.001)(\mathrm{B})$. Group A includes following benefit items: cancer, tuberculosis and disability. Group B includes following benefit items: long-term hospitalization, rarely incurable, secondary poor, secondary poor with disability, hemodialysis, and severely ill. 
Table 3. Univariate and multivariate analysis of factors associated with in-hospital mortality in total patients and subjects with health insurance benefit items

\begin{tabular}{|c|c|c|c|c|}
\hline \multirow{2}{*}{ Factor } & \multicolumn{2}{|c|}{ Univariate analysis } & \multicolumn{2}{|c|}{ Multivariate analysis } \\
\hline & $\mathrm{HR}(95 \% \mathrm{Cl})$ & P-value & HR $(95 \% \mathrm{Cl})$ & P-value \\
\hline \multicolumn{5}{|l|}{ Total patients } \\
\hline Patients without health insurance benefit items & $2.805(1.993-3.947)$ & $<0.001$ & $2.794(1.980-3.941)$ & $<0.001$ \\
\hline SOFA score ${ }^{a}$ & $1.175(1.112-1.241)$ & $<0.001$ & $1.138(1.068-1.212)$ & $<0.001$ \\
\hline Age $\geq 65$ yr & $1.465(0.958-2.242)$ & 0.078 & & \\
\hline \multicolumn{5}{|l|}{ Patients with health insurance benefit items ${ }^{b}$} \\
\hline Group A & $3.951(2.583-6.045)$ & $<0.001$ & $3.904(2.533-6.039)$ & $<0.001$ \\
\hline SOFA score ${ }^{a}$ & $1.107(1.028-1.192)$ & 0.007 & & \\
\hline
\end{tabular}

Statistical significance was tested by univariate Cox proportional hazards models and multivariate Cox hazards models.

HR: hazard ratio; $\mathrm{Cl}$ : confidence interval; SOFA: Sequential Organ Failure Assessment.

${ }^{a}$ All clinical data was calculated or checked from; ${ }^{\circ}$ Patients were divided into two groups (group A includes following benefit items: cancer, tuberculosis and disability. Group B includes following benefit items: long-term hospitalization, rarely incurable, secondary poor, secondary poor with disability, hemodialysis, and severely ill).

suggest that the health insurance benefit range of this policy should be adjusted so that ventilated patients can effectively receive quality healthcare services without facing any financial burden because universal healthcare coverage is also a key concern of critically ill patients $[4,11]$. Furthermore, it would be necessary for critical care physicians to consider the benefit items and out-of-pocket medical expenditure of patients when discussing future treatment planning with their families or surrogates.

In further analyses of patients with benefit items, clinical characteristics and outcomes differed according to benefit items under this policy. Although patients with group A benefit items received significant financial benefits, reflected by a significantly lower percentage of out-of-pocket medical expenditure relative to total medical expenditure than patients with group B benefit items, they had significantly more severe illness and accompanying organ failure at ICU admission and higher in-hospital mortality. Our study indicates that simply reducing out-of-pocket expenditure by implementing this policy would not markedly affect the survival of these patients.

In our study, we tried to investigate whether the NHI benefit extension policy and benefit items under this policy were useful prognostic indicators. For assessing prognosis, various clinical and demographic indicators should be considered, however, we could not comprehensively do this because our study had a single-center retrospective design and included a small number of patients. Furthermore, the present study indicates in-hospital mortality differed according to benefit items and associated benefit rule. In particular, the clinical outcomes of patients with the benefit item "cancer (solid and hematologic malignancies)" differ depending on the type and stage. Therefore, additional studies including a large number of patients are needed to determine whether each benefit item under this policy would be a valuable prognostic indicator in critical ill ventilated patients.

Our study has several limitations. First, we hypothesized the family support and socioeconomic status of each patient would be associated with clinical outcomes, however, we could not evaluate this because our study had an observational retrospective design. Second, we investigated the effect of the NHI benefit extension policy on patients requiring ventilation due to respiratory diseases. However, the relationships between this policy and clinical outcomes may differ in patients requiring ventilation for other reasons. Further studies of heterogeneous populations are needed to evaluate the effect of this policy on clinical outcomes. Finally, this study was performed in one hospital, and the sample size was small; therefore, our results may not represent the general situation in Korea. However, considering that our institution is one of the best-equipped hospitals in our country, and that the health insurance benefit expansion policy and associated benefit rules apply across Korea, the situation in other Korean hospitals is likely similar to that in our hospital.

In conclusion, analysis of the Korean NHI coverage benefit extension policy in ventilated patients showed that this policy increased the use of medical resources and associated improved in-hospital mortality of NHI beneficiaries. Furthermore, the percentage of out-of-pocket medical expenditure relative to total medical expenditure and in-hospital mortality differed according to benefit items under this policy. Large- 
scale multicenter studies are required to determine the effect of this policy on clinical outcomes of patients requiring ventilator care due to reasons other than respiratory diseases.

\section{CONFLICT OF INTEREST}

Kwangha Lee is an editorial board member of the journal but was not involved in the peer reviewer selection, evaluation, or decision process of this article. No other potential conflicts of interest relevant to this article were reported.

\section{ACKNOWLEDGMENTS}

This work was supported by Department of Biostatistics, Biomedical Research Institute, Pusan National University Hospital. And, this work was supported by clinical research grant from Pusan National University Hospital in 2021.

\section{ORCID}

Wanho Yoo

https://orcid.org/0000-0002-2344-0231

Saerom Kim https://orcid.org/0000-0002-5093-1503

Soohan Kim https://orcid.org/0000-0002-4549-0862

Eunsuk Jeong https://orcid.org/0000-0002-6724-3942

Kwangha Lee

\section{AUTHOR CONTRIBUTIONS}

Conceptualization: WY, KL. Data curation: all authors. Formal analysis: WY, KL. Funding acquisition: KL. Methodology: WY, KL. Project administration: WY, KL. Visualization: WY, KL. Writing-original draft: WY, KL. Writing-review \& editing: KL.

\section{REFERENCES}

1. Ministry of Health and Welfare. Social security system in Korea [Internet]. Sejong: Ministry of Health and Welfare; 2021 [cited 2021 May 25]. Available from: http://www.mohw.go.kr/eng/pl/ pl0101.jsp?PAR_MENU_ID=1003\&MENU_ID=100324.

2. Lee WY, Shaw I. The impact of out-of-pocket payments on health care inequity: the case of National Health Insurance in South Korea. Int J Environ Res Public Health 2014;11:7304-18.

3. Kim JA, Yoon S, Kim LY, Kim DS. Towards actualizing the value potential of Korea Health Insurance Review and Assessment (HIRA) data as a resource for health research: strengths, limitations, applications, and strategies for optimal use of HIRA data. J Korean Med Sci 2017;32:718-28.

4. An J, Kim S. Medical cost trends under national health insurance benefit extension in Republic of Korea. Int J Health Plann Manage 2020;35:1351-70.

5. Acute Respiratory Distress Syndrome Network; Brower RG, Matthay MA, Morris A, Schoenfeld D, Thompson BT, et al. Ventilation with lower tidal volumes as compared with traditional tidal volumes for acute lung injury and the acute respiratory distress syndrome. N Engl J Med 2000;342:1301-8.

6. Bahk J, Kang HY, Khang YH. Trends in life expectancy among medical aid beneficiaries and National Health Insurance beneficiaries in Korea between 2004 and 2017. BMC Public Health 2019;19(1):1137.

7. Welfare services at a glance [Internet]. Seoul: Ministry of Health and Welfare and Korea Social Security Information Service; 2021 [cited 2021 May 25]. Available from: https://www.bokjiro. go.kr/ssis-teu/index.do.

8. Knaus WA, Draper EA, Wagner DP, Zimmerman JE. APACHE II: a severity of disease classification system. Crit Care Med 1985;13:818-29.

9. Vincent JL, Moreno R, Takala J, Willatts S, De Mendonça A, Bruining H, et al. The SOFA (Sepsis-related Organ Failure Assessment) score to describe organ dysfunction/failure: on behalf of the Working Group on Sepsis-Related Problems of the European Society of Intensive Care Medicine. Intensive Care Med 1996;22:707-10.

10. Charlson ME, Pompei P, Ales KL, MacKenzie CR. A new method of classifying10 prognostic comorbidity in longitudinal studies: development and validation. J Chronic Dis 1987;40:373-83.

11. Kutzin J, Sparkes SP. Health systems strengthening, universal health coverage, health security and resilience. Bull World Health Organ 2016;94:2. 